\title{
IDOSOS DISTANCIADOS DA SOCIEDADE E O SENTIDO DA VIDA: CUIDADOS DIRECIONADOS AO RISCO DE SÍNDROME DO IDOSO FRÁGIL
}

\author{
Maria Helena Lenardt' \\ ORCID:0000-0001-8309-4003
}

Karina Silveira De Almeida Hammerschmidt'

ORCID: 0000-0002-7140-3427

Márcia Marrocos Aristides Barbiero'

ORCID: 0000-0002-1567-3641

Ligia Carreira"

ORCID: 0000-0003-3891-4222

Clóris Regina Blanski Grden"'

ORCID: 0000-0001-6169-8826

Susanne Elero Betiolli'

ORCID: 0000-0003-4469-4473

Denise Faucz Kletemberg ${ }^{\text {IV }}$

ORCID: 0000-0001-7289-7900

'Universidade Federal do Paraná Curitiba, Paraná, Brasil.

"Universidade Estadual de Maringá. Maringá, Paraná, Brasil.

"'Universidade Estadual de Ponta Grossa. Ponta Grossa, Paraná, Brasil.

Iv Universidade Positivo. Curitiba, Paraná, Brasil.
Autor Correspondente:

Maria Helena Lenardt

E-mail: curitiba.helena@gmail.com

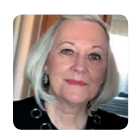

Como citar: Lenardt MH, Hammerschmidt KSA, Barbiero MMA, Carreira L, Grden CRB, Betiolli SE, Kletemberg DF. Idosos Distanciados da Sociedade e o Sentido Da Vida: Cuidados Direcionados ao Risco de Síndrome do Idoso Frágil. In: Santana RF (Org.). Enfermagem gerontológica no cuidado do idoso em tempos da COVID 19. Brasilia, DF: Editora ABen; 2021. 171 p. (Serie Enfermagem e Pandemias, 5). https://doi.org/10.51234/aben.21.e05.c10

\section{INTRODUÇÃO}

No âmbito de alcance profissional em gerontologia é necessário promover o envelhecimento saudável, gerenciando os fatores e as condições de risco, que levam a quadros de dependência e perda de autonomia. Essas são condições que podem estar relacionadas à síndrome da fragilidade física, importante preditor de agravos à saúde de idosos.

A síndrome da fragilidade física no idoso é uma condição clínica na qual há aumento da vulnerabilidade do indivíduo para desenvolver doenças, incapacidades, quedas e até mesmo a morte, quando exposto a agente estressor. Ela envolve fatores biológicos, físicos, psicológicos, cognitivos, socioeconômico e ambientais ${ }^{(1-2)}$.

Recentemente, a International Conference of Frailty and Sarcopenia Research (ICFSR) apresentou as diretrizes internacionais da prática clínica para identificação e gerenciamento da fragilidade física, com base em evidências. As recomendações concentram-se nos aspectos clínicos e práticos do atendimento aos idosos com fragilidade e promovem cuidados centrados na pessoa, por meio de plano abrangente de gestão da fragilidade, que inclui: redução da polifarmácia, manejo da sarcopenia, atividade física multicomponente, suplementação calórico-proteica, causas tratáveis da perda de peso e da fadiga/exaustão, além de apoio social(2).

No que diz respeito ao componente fadiga / exaustão, o manejo da fragilidade se faz necessário diante do elevado quantitativo de idosos com sintomas depressivos, uma vez que estes possuem relação com o componente. Os sintomas se manifestam sutilmente, com disforia e sintomas somáticos, são frequentemente associados aos traços de depressão, com desfechos negativos à saúde do idosos ${ }^{(3)}$.

Uma revisão sistemática com metanálise foi desenvolvida com o objetivo de estimar a prevalência de sintomatologia depressiva em idosos brasileiros residentes na comunidade. Foram incluídas 33 publicações, que totalizaram 39.431 idosos, e os resultados indicaram prevalência de $21,0 \%$ 
dos idosos com sintomatologia depressiva, com variação entre $7,1 \%$ em estudo realizado no sul do país e $39,6 \%$ em estudo realizado no nordeste ${ }^{(3)}$.

Frente à pandemia da Covid-19 observa-se a possibilidade de agravamento dessa sintomatologia depressiva, que frequentemente está associada à fadiga e exaustão, bem como da condição de fragilidade do idoso. $O$ momento atual evidenciou a necessidade de bloqueios, distanciamento e isolamento social para mitigar a disseminação do novo coronavírus (SARS-CoV-2), na tentativa de proteger os idosos e os sistemas de saúde sobrecarregados. Embora estas sejam medidas protetivas, é preciso considerar as repercussões para a saúde física e mental dos idosos ${ }^{(4)}$.

Nesse contexto, recomenda-se a preservação da saúde mental do idoso com estratégias que valorizam o Sentido da Vida, principalmente para aqueles com risco de síndrome do idoso frágil. A busca do Sentido da Vida pode ser compreendida como a força motivadora do ser humano. Alguns estudos identificaram a influência do Sentido da Vida para a saúde dos idosos e o apontam como alvo intervencionista promissor, além de ser fator protetivo que auxilia no enfrentamento de situações adversas e potencializa os níveis de saúde ${ }^{(5-6)}$.

\section{OBJETIVO}

Propor cuidados gerontológicos direcionados ao risco de síndrome do idoso frágil fundamentados na busca do sentido da vida em tempo de distanciamento da sociedade.

\section{MÉTODO}

Este capítulo traz um texto teórico-reflexivo, que foi alicerçado na temática fragilidade associada à linha do fenótipo da fragilidade física de Linda Fried e colaboradores, na Teoria da Logoterapia e Análise Existencial de Victor E. Frankl, nos diagnósticos do NANDA-I (7), e em artigos científicos sobre a temática.

\section{RESULTADOS E DISCUSSÕES}

\section{Risco de síndrome do idoso frágil e a proteção pela busca do Sentido da Vida}

Neste cenário de pandemia é preciso dar atenção especial para o idoso com "Risco de Síndrome do Idoso Frágil"(7), pois esta situação de ameaça pode ser a alavanca para torná-los mais sensíveis às novas necessidade. A fragilidade no idoso é uma síndrome de declínio espiral de energia, embasada por tripé de alterações relacionadas ao envelhecimento, composto por sarcopenia, desregulação neuroendócrina e disfunção imunológica.

Os idosos portadores dessa tríade estão propensos à redução acentuada da massa muscular e a estado inflamatório crônico que, se associados aos fatores extrínsecos como a incidência de doenças agudas ou crônicas, a imobilidade, a redução da ingesta alimentar e outros, levam a ciclo vicioso de redução de energia e aumento da dependência e susceptibilidade aos agressores ${ }^{(8)}$.

De acordo com os marcadores do fenótipo da fragilidade, o idoso fragilizado classificado como pré-frágil é aquele que apresenta um a dois marcadores, enquanto aqueles que apresentam três ou mais marcadores são classificados como frágeis ${ }^{(1)}$. O fenótipo é composto por cinco marcadores biológicos passíveis de mensuração: perda de peso não intencional, autorrelato de fadiga/exaustão, diminuição da força de preensão manual, diminuição das atividades físicas e redução da velocidade da marcha.

Entre os idosos com Diagnóstico de Enfermagem "Risco de Síndrome do Idoso Frágil"(7) se observa, sutilmente, algum desses marcadores do fenótipo da fragilidade. O distanciamento da sociedade, como medida preventiva, pode desencadear complicações adicionais nesses marcadores, acentuando o risco e como desfecho a evolução da condição de fragilidade. Nesse contexto as complicações adicionais estão relacionadas ao convívio social, que essa situação tem potencial para gerar sintomas depressivos, uma vez que eles se veem mais longe do convívio social e familiar. 
A fragilidade e os sintomas depressivos são apontados na literatura como bidirecionais e configuram-se como condições comuns entre os idosos ${ }^{(9)}$. Um importante estudo constatou que, em 8.023 indivíduos com fragilidade, a prevalência de depressão foi de $38,6 \%$. Os idosos frágeis apresentavam quatro vezes mais propensão a ter depressão, quando comparados aos não frágeis ou robustos ${ }^{(9)}$.

As duas condições estão associadas aos fatores de risco, os quais podem estar compartilhados. Alterações fisiológicas decorrentes do processo de envelhecimento podem ocasionar sintomas de fadiga e lentidão na marcha, bem como a diminuição de atividade física e a perda de peso, déficits cognitivos e funcionais ${ }^{(10)}$.

Esses déficits podem ser observados tanto para aqueles idosos cujo único contato social está fora de casa, em atividades em centros comunitários, reuniões com amigos e religiosas, quanto para aqueles que não têm familiares ou amigos próximos e dependem do apoio de serviços voluntários ou assistência social. Essa desconexão social coloca os idosos em maior risco de ansiedade e sintomas depressivos ${ }^{(11,4)}$.

Os idosos com sintomas depressivos tendem a interpretar negativamente os eventos da vida, pressupondo resultados desfavoráveis e assumindo comportamentos que se relacionam aos sentimentos pessoais de inadequação, desesperança, sensação de vazio existencial ou falta de sentido da vida. Esses sentimentos, regularmente, levam os idosos ao declínio da saúde física e a um quadro de depressão ${ }^{(12)}$.

De acordo com a teoria elaborada por Viktor Frankl, denominada Logoterapia e Análise Existencial, a busca por Sentido da Vida seria uma necessidade cotidiana, gerada por uma motivação intrínseca ao ser e se refere ao interesse contínuo do ser humano por um significado para a sua vida ${ }^{(13)}$. Existem duas dimensões principais no Sentido da Vida: a busca pelo sentido e a presença de sentido. O primeiro refere-se ao grau de envolvimento de uma pessoa na busca de sentido na vida, enquanto o segundo refere-se ao grau de percepção individual em relação à própria vida como significativa(14).

O Sentido da Vida está relacionado à crença de que a vida do indivíduo é plena de significados, que existe um propósito, uma direção, uma razão particular para a existência. Todas as vezes que o indivíduo alcança suas ambições, novos objetivos de vida tendem a surgir, deixando-o sempre orientado para o futuro, buscando novas realizações e novos significados para a existência, enquanto a sua ausência é capaz de gerar um estado de apatia, desânimo e sintomas depressivos ${ }^{(15)}$.

Indivíduos que têm interpretações significativas da vida com a presença de objetivos e propósito tendem a ter melhor bem-estar. O Sentido da Vida foi identificado como importante para a qualidade de vida dos idosos, não apenas como um componente significativo do bem-estar, mas como um recurso de enfrentamento com importância clínica para a saúde física e mental ${ }^{(16)}$.

Um maior nível de Sentido da Vida foi associado à maior percepção de felicidade, melhor estado de saúde (menor nível de fragilidade, menos doenças crônicas e limitações de atividades, melhor autoavaliação) (16). A experiência do SV tem um fator protetivo, uma vez que medeia parcialmente o otimismo, influencia a função física, o bem estar e promove resistência contra o estresse das dificuldades vividas pelos idosos ${ }^{(16,15)}$.

Reconhece-se que uma fonte importante para o Sentido da Vida em idosos são os relacionamentos. Os indivíduos encontram Sentido na Vida mediante sua relação com o mundo ${ }^{(13)} \mathrm{e}$, por vezes, dependem dessas relações como suporte para a vida. $\mathrm{O}$ ambiente social proporciona apoio por meio das relações familiares e de amizades, as oportunidades de nutrição, alianças confiáveis que são importantes ${ }^{(17)}$.

Destaca-se a importância da convivência social como fonte de suporte relacionada ao senso de bem-estar da pessoa idosa. Os laços sociais podem amortecer eventos estressantes e adversos da vida, combatendo o aparecimento de sintomas depressivos ou atenuando seus efeitos negativos ${ }^{(11)}$.

Estudo apontou o aumento na qualidade de vida, da longevidade e no funcionamento físico de idosos que mantêm uma vida social intensa, sugerindo que relacionamentos sociais são fatores importantes para o bem-estar físico e mental na velhice ${ }^{(18)}$. Enquanto a adversidade psicossocial contribui para as mudanças fisiológicas, aumentando a suscetibilidade à depressão em idosos vulneráveis e pré-frágeis ${ }^{(19)}$. 


\section{Cuidados gerontológicos direcionados ao “Risco de Síndrome do Idoso Frágil”(7):a busca por Sentido da Vida dos idosos em tempo da pandemia COVID-19}

Entre os Diagnósticos de Enfermagem (DE) da Taxonomia NANDA-I ${ }^{(7)}$, encontram-se os de "Risco de Síndrome do idoso frágil" e "Síndrome do Idoso Frágil" (SIF). Esses DE foram incluídos na versão da NANDA-I de 2015-2017, a partir de resultados de pesquisas no âmbito hospitalar e comunitário no contexto do cuidado ao idoso, por um grupo de pesquisadores da Universidad de Castilla La Mancha, na Espanha ${ }^{(20)}$. Considera-se, portanto, que se tratam de diagnósticos novos, pouco explorados e que carecem de pesquisas e de uso recorrente na realidade brasileira.

O “Risco de Síndrome do Idoso Frágil” é definido como: "Suscetibilidade a estado dinâmico de equilíbrio instável que afeta o idoso que passa por deterioração em um ou mais domínios de saúde (físico, funcional, psicológico ou social) e leva ao aumento da suscetibilidade a efeitos de saúde adversos, em particular a incapacidade $^{\prime \prime(7: 266)}$. Considerando esse $\mathrm{DE}$, com ênfase nas consequências geradas pela pandemia COVID-19, destaca-se a preocupação com o distanciamento social e sintomas depressivos, e com fatores de riscos, alinhados à população de risco e às condições associadas relevantes para o cuidado gerontológico de Enfermagem, conforme destacados no quadro 1.

Quadro 1 - Diagnóstico de Enfermagem "Risco de Síndrome do Idoso Frágil"(7) com ênfase em consequências da pandemia COVID-19 (distanciamento da sociedade e sintomas depressivos):fatores de risco, população de risco e condições associadas.

\begin{tabular}{|l|l|l|}
\hline Fatores de risco & População de risco & Condições associadas \\
\hline & Baixo nível educacional & Alteração na função cognitiva \\
& Desfavorecido economicamente & Anorexia \\
Ansiedade & História de quedas & Obena crônica \\
Depressão & Idade $>70$ anos & Perda não intencional de $>4,5 \mathrm{~kg}$ em 1 ano \\
Exaustão & Morar só & Perda não intencional de 25\% do peso corporal em 1 ano \\
Isolamento social & Sexo feminino & Redução da concentração sérica de 25-hidroxivitamina D \\
& Viver em espaço limitado & Resposta inflamatória suprimida \\
& Vulnerabilidade social & Sarcopenia \\
\hline
\end{tabular}

Fonte: NANDA-I, 2018.

No Diagnóstico de Enfermagem "Risco de Síndrome do Idoso Frágil"(7) são encontrados fatores de riscos definidos como influências que elevam a fragilidade dos indivíduos a determinado evento não saudável, sobretudo no período pandêmico, como: ansiedade, depressão, exaustão e isolamento social.

Considera-se a ansiedade como sentimento vago e incômodo de desconforto ou temor, acompanhado por resposta autonômica (a fonte é frequentemente não específica ou desconhecida para o indivíduo); sentimento de apreensão causado pela antecipação de perigo. É um sinal de alerta que chama a atenção para um perigo iminente e permite ao indivíduo tomar medidas para lidar com a ameaça ${ }^{(7: 614)}$.

A depressão é o estado subjetivo no qual um indivíduo vê alternativas limitadas, ou não vê alternativas ou escolhas pessoais disponíveis, e é incapaz de mobilizar energias em benefício próprio(7:510). Considera-se exaustão a sensação opressiva e prolongada de exaustão e capacidade diminuída de realizar trabalho físico e mental no nível habitual (7:419). Por sua vez, o isolamento social envolve a solidão sentida pelo indivíduo, percebida como imposta por outros e como um estado negativo ou ameaçador ${ }^{(7: 913)}$.

Desta forma, destaca-se no Quadro 2 os cuidados gerontológicos de Enfermagem relevantes na busca do sentido da vida dos idosos em tempo da pandemia COVID-19, com ênfase nos fatores de risco elencados como prioritários no DE "Risco de Síndrome do Idoso Frágil"(7):ansiedade, depressão, exaustão e isolamento social. Esses cuidados poderão ser viabilizados mediante teleatendimento, consulta de Enfermagem virtual, encontros individuais e grupais de forma remota, mensagens instantâneas, disponibilização de gerontotecnologias educacionais (folder, cartilha, planos/programações). 
Quadro 2 - Cuidados Gerontológicos de Enfermagem alinhados ao referencial do Sentido da Vida, direcionados pelos fatores do Diagnóstico de Enfermagem "Risco de Síndrome do Idoso Frágil" em tempo da pandemia COVID-19: ansiedade, depressão, exaustão e isolamento social(7)

Fatores de $\operatorname{risco}^{(7)}$

Ansiedade $^{(7)}$

Depressão(7) $^{(7)}$

Exaustão $^{(7)}$
Cuidados Gerontológicos de Enfermagem em tempo de pandemia COVID-19, alinhados ao referencial do Sentido da Vida

- Reduzir estresse por mudança, ocasionado pela pandemia COVID, sobretudo em relação ao distanciamento social, por meio de monitoramento telefônico e escuta ativa.

- Apoiar a tomada de decisão do idoso, mediante consulta de Enfermagem realizada por teleatendimento, com orientações e estímulos a comportamentos saudáveis.

- Realizar biofeedback com o idoso e seus familiares, sobre o sentido da vida, considerando, em especial, o momento pandêmico, utilizando recursos tecnológicos de chamada virtual.

- Promover melhorias no sono do idoso, com inclusão de atividades que possibilitem reflexão, autoconhecimento, relaxamento, orientadas de forma virtual.

- Desenvolver orientação antecipada, mediante consulta de Enfermagem com idoso e seus familiares, com foco nos sinais e sintomas da COVID-19, possibilitando busca precoce por atenção à saúde, caso seja necessário. - Promover e incentivar ao exercício físico, apresentando ao idoso e seus familiares atividades diárias a serem realizadas, com cronograma pactuado em consulta de Enfermagem.

- Realizar visita / consulta virtual ao idoso, orientando sobre aspectos que envolvem o ambiente seguro neste momento de pandemia COVID-19.

- Organizar grupo de apoio para interação social virtual do idoso, com encontros periódicos coordenados.

- Oferecer possibilidades de terapia recreacional, de relaxamento e gerontotecnologias educacionais de forma virtual (com folder, vídeo ou cartilha)

- Estimular atividade artísticas, que melhoram a capacidade motora e apoiam a realização da vida diária, enfatizam o bem-estar e a autoimagem;

- Estimular a prática das atividades manuais e de leituras capazes de proporcionar estímulo à memória e atenção, além de satisfação de ser útil e capaz.

- Realizar acompanhamento do comportamento, mediante teleconsulta, realizada de forma periódica com o idoso.

- Orientar familiares para atenção aos sinais e indícios de comportamento suicida, visando à prevenção e intervenção precoce.

- Estimular a reflexão sobre a espiritualidade e/ou religiosidade colaborando com o encontro do propósito e o significado da existência humana.

- Motivar o idoso a buscar o Sentido da Vida, resgatando sua trajetória e fortalecendo afetos e atitudes positivas de suas vivências.

- Reconhecer o sistema de crenças que possam ajudar o idoso no enfrentamento de questões do cotidiano e a perceber possibilidades de soluções a serem realizadas.

- Fortalecer idoso e seus familiares sobre a esperança de enfrentamento da pandemia COVID-19, ressignificando os aprendizados deste período.

- Estimular o sentimento de gratidão e orgulho pelas realidades do passado como parte da existência e que auxiliam no otimismo sobre o futuro.

- Possibilitar a Reestruturação Cognitiva de forma remota, refletindo em conjunto com o idoso a importância de modificar pensamentos disfuncionais, distorções cognitivas e crenças limitantes.

- Promover momento de grupo familiar virtual, no qual possam ser refletidos sentimentos e maneiras como está sendo vivida a pandemia.

- Realizar estabelecimento de metas mútuas para este tempo de pandemia, com chamada de vídeo que envolva idoso e seus familiares/cuidadores.

- Refletir junto com o idoso, em ação grupal online, a complexidade envolvida neste momento pandêmico, reforçando a busca pela vivência de valores e significados de vida.

- Promover Terapia de Reminiscências, de forma individual ou coletiva (virtual / semanal), auxiliando na revisão cronológica da vida, analisando os eventos mais marcantes e vislumbrando a criação de registro de vida (por exemplo livro), incentivando a partilha das experiências.

- Realizar monitoramento do estado nutricional do idoso, mediante investigação clínica realizada em consulta de enfermagem virtual.

- Promover orientações sobre exercícios físicos, dieta e atividades saudáveis durante o período de distanciamento social, com envio de materiais digitais para telefones celulares e/ou email.

- Incentivar atividades a partir de interesses pessoais, que façam sentido para o idoso, estimulem a autonomia e capacidade de tomar decisões.

- Disponibilizar informações sobre autocuidado, de forma digital para idosos e seus familiares/cuidadores.

- Monitorar a dor dos idosos, mediantes entrevista realizada em consulta de Enfermagem por teleatendimento.

- Supervisionar o nível de fadiga dos idosos, mediante consulta de Enfermagem virtual, com apoio e acompanhamento dos familiares/cuidadores. 
Fatores de risco $^{(7)}$

Isolamento social ${ }^{(7)}$
Cuidados Gerontológicos de Enfermagem em tempo de pandemia COVID-19, alinhados ao referencial do Sentido da Vida

Promover o vínculo do idoso com seus familiares, mediante encontros coletivos que valorizem a reflexão acerca da busca do sentido da vida.

- Fomentar o apoio familiar, mediante consulta de Enfermagem mensal, que possibilite momento individual e familiar. - Pactuar com idoso e seus familiares/cuidadores, corresponsabilidades a serem realizadas neste período pandêmico, visando qualidade de vida e comportamentos saudáveis.

- Possibilitar ao idoso socialização, mediantes encontros grupais de forma virtual, utilizando ferramentas tecnológicas de comunicação.

- Auxiliar o idoso a perceber tarefas que podem ser realizadas no presente e ampliar a perspectiva de planos para futuro.

- Estimular a realização de terapias, conforme as preferências do idoso, mediante orientação de forma virtual.

- Elaborar junto ao idoso, programação de atividades a serem realizadas por ele e seus familiares, envolvendo aspectos para melhoria da comunicação, lazer e distração.

- Realizar a Terapia do Espelho, na qual o idoso ao ver sua própria imagem de forma virtual exercita o autoconhecimento.

- Aconselhar o idoso sobre os sentimentos, em consulta de enfermagem virtual específica para este fim, refletindo sobre as percepções e significados, promovendo estímulo para controle do humor.

- Monitorar em consultas de Enfermagem periódicas, por teleatendimento, a utilização dos medicamentos contínuos, conferindo junto com o idoso e seus familiares receitas médicas.

- Estimular os idosos, familiares e cuidadores a promoverem visitas virtuais, possibilitando interação e socialização com segurança.

A velhice é a última fase da vida e neste momento infelizmente verifica-se a perda de amigos, papel profissional e separação familiar, o que pode gerar diminuição do contato social e solidão. Esses fatores são reforçados com a pandemia COVID-19, na qual o distanciamento social foi recomendação padrão(21).

Além disso, os estigmas em relação aos idosos, exacerbados principalmente durante a pandemia, podem afetá-los negativamente, produzindo sentimentos de inutilidade social e suscitando pessimismo em relação ao futuro. Sendo assim, o isolamento social e desesperança repercutem na saúde mental, podendo estar associados à depressão e ansiedade ${ }^{(22)}$.

Estratégias como o engajamento em atividades estimulantes, com práticas motivacionais para a busca do Sentido da Vida, em tempo da pandemia COVID-19, podem promover o bem-estar e autoconceito positivo, proporcionando reconhecimento das emoções e dos acontecimentos no momento presente (Valor vivencial), favorecendo o aumento do nível de satisfação dos idosos e a vivência dos valores que efetivam o Sentido da Vida.

Neste momento pandêmico, ações promovidas de forma virtual são seguras, considerando a necessidade de distanciamento social. Possibilitam a participação do idoso em ações individuais e coletivas, afastando o isolamento social, promovendo integração, aumentando a autoestima e melhorando o relacionamento com familiares. Essas ações pactuadas, com orientação profissional, estimulam a criatividade e a cooperação mútua, com propósito para além da convivência, melhorando a memória, a atenção e a socialização dos idosos (Valor criativo).

Considerar os aspectos cognitivos como método de atribuição dos eventos da vida, das habilidades interpessoais e da percepção do Sentido da Vida são fundamentais para a experiência dos valores ${ }^{(23)}$. Acompanhar e estimular o idoso por meio de atividades que busquem soluções para as questões vividas, evocando recordações e significados, incentivando a cooperação, a remotivação, a valorização da autoestima, a alegria e o otimismo podem promover a proteção pela busca do sentido de vida (Valor atitudinal).

\section{Limitações do estudo}

O estudo não se propõe a esgotar a reflexão teórica e conceitual acerca dos cuidados gerontológicos de Enfermagem aos idosos em "Risco de Síndrome do Idoso Frágil"(7), em situações de distanciamento social consequente da pandemia COVID-19; mas tem intenção de provocar reflexões alinhadas ao referencial do Sentido da Vida, podendo apontar diretrizes que possam sustentar debates e ações em gerontologia. 


\section{Contribuições para a Área}

Este artigo de reflexão contribui tanto aos processos formativos quanto às instituições de saúde, considerando trazer cuidados gerontológicos para o "Risco de Síndrome do Idoso Frágil"(7). Nessa perspectiva, considera-se que as estratégias que valorizam o Sentido da Vida dos idosos, ora expostas, são valiosas à atuação da Enfermagem, diante dos idosos em situação de fragilidade e que se encontram em distanciamento da sociedade. Isso posto, a contribuição deste estudo se encontra nas possibilidades de cuidados gerontológicos de Enfermagem que favoreçam o aumento do nível de satisfação dos idosos e a vivência dos valores que efetivam o Sentido da Vida, em tempos de pandemia.

\section{CONSIDERAÇÕES FINAIS}

O cuidado gerontológico de Enfermagem desenvolvido com ênfase na busca do Sentido da Vida do idoso, principalmente neste momento de pandemia COVID-19, promove a integralidade do cuidado, permite a identificação precoce dos fatores de risco para fragilidade e possibilita inovar nas práticas de Enfermagem. A proposta de cuidados de enfermagem, desenvolvidas frente às necessidades emergentes em tempo de pandemia, re-significam os momentos vividos, fortalecendo o idoso como centralidade do cuidado, respeitando sua história, necessidades, desejos e responsabilidades.

A Enfermagem, além de suas atividades presenciais heróicas, atuando durante a pandemia na linha de frente do cuidado, oportuniza inovação nos cuidados gerontológicos por "conexão à distância", apropriando-se de recursos tecnológicos, tais como redes e mídias sociais, canais de comunicação e aplicativos para smartphone. Esses meios são contribuições relevantes, pois possibilitam a aproximação e o acompanhamento de idosos que se encontram em distanciamento da sociedade.

\section{REFERÊNCIAS}

1. Fried L, Tangen CM, Walston J, Newman AB, Hirsch C, Gottdiener J, et al. Frailty in older adults: evidence for a phenotype. J Gerontol A Biol Sci Med Sci. 2001;56A(3):146-56. https://doi.org/10.1093/gerona/56.3.m146

2. Dent E, Morley JE, Cruz-Jentoft AJ, Woodhouse L, Rodríguez-Manã L, Fried L, et al. Physical frailty: ICFSR International Clinical Practice Guidelines for Identification and Management. J Nutrit Health Aging. 2019;23(9):771-87. https://doi. org/10.1007/s12603-019-1273-z

3. Meneguci J, Meneguci CAG, Moreira MM, Pereira KR, Tribess S, Sasaki JE, et al. Prevalência de sintomatologia depressiva em idosos brasileiros: uma revisão sistemática com metanálise. J Bras Psiquiatr. 2019;68(4):221-30. https://doi. org/10.1590/0047-2085000000250

4. Armitage R, Nellums LB. COVID-19 and the consequences of isolating the elderly. Lancet Public Health. 2020;5(5):e256. https://doi.org/10.1016/S2468-2667(20)30061-X

5. Musich S, Wang SS, Kraemer S, Hawkins K, Wicker E. Purpose in life and positive health outcomes among older adults. Population Health Management. 2018;21(2):139-47. https://doi.org/10.1089/pop.2017.0063

6. Sampaio LANPC, Ayres JRCM. Critique of the concept of motivation and its implications for healthcare practices. Philosop Ethics Human Med. 2019;14(1):1-10. https://doi.org/10.1186/s13010-019-0083-6

7. Herdman TH, Kamitsuru S. NANDA - Internacional. Diagnóstico de Enfermagem da NANDA: definições e classificação 20182020. 11 ed. Porto Alegre: Artmed; 2018.

8. Cesari M, Prince M, Thiyagarajan JA, Carvalho IA, Bernabei R, Chan P, et al. Frailty: an emerging public health priority. J Am Med Dir Assoc. 2016;17(3):188-92. https://doi.org/10.1016/j.jamda.2015.12.016

9. Soysal P, Veronese N, Thompson T, Kahl KG, Fernandes BS, Prina AM, et al. Relationship between depression and frailty in older adults: a systematic review and meta-analysis. Ageing Res Rev. 2017;36:78-87. https://doi.org/10.1016/j.arr.2017.03.005

10. Lohman M, Dumenci L, Mezuk B. Depression and frailty in late life: evidence for a common vulnerability. J Gerontol B Psychol Sci Soc Sci. 2016;71(4):630-40. https://doi.org/10.1093/geronb/gbu180 
11. Herbolsheimer F, Ungar N, Peter R. Why is social isolation among older adults associated with depressive symptoms? the mediating role of out-of-home physical activity. Int J Behav Med. 2018;25:649-57. https://doi.org/10.1007/ s12529-018-9752-x

12. Aquino TAA, Veloso VG, Aguiar AA, Serafim TDB, Pontes AM, Pereira GA, et al. Questionário de sentido de vida: evidências de sua validade fatorial e consistência interna. Psicol Cienc 2015;35(1):4-19. https://doi.org/10.1590/1982-3703001332012

13. FrankI VE. Em busca de sentido: um psicólogo no campo de concentração. 31 ed. Rio Grande do Sul: Sinodal; 2011.

14. Chuí RCF. The role of meaning in life for the quality of life of community-dwelling Chinese elders with low socioeconomic status. Gerontol Geriatric Med. 2018;4:1-8. https://doi.org/10.1177/2333721418774147

15. Damásio BF, Koller SH. Meaning in life questionnaire: adaptation process and psychometric properties of the Brazilian version. Rev Latino-Am Psicol. 2015;47(3):185-95. https://doi.org/10.1016/j.rlp.2015.06.004

16. Zhang D, Chan DC, Niu L, Liu H, Zou D, Chan ATY, et al. Meaning and its association with happiness, health and healthcare utilization: a cross-sectional study. J Affect Disord. 2018;227:795-02. https://doi.org/10.1016/j.jad.2017.11.082

17. Duppen D, Van der Elst MCG, Dury S, Lambotte D, De Donder L. The social environment's relationship with frailty: evidence from existing studies. J Applied Gerontol. 2019;38(1):3-26. https://doi.org/10.1177/0733464816688310

18. Wichmann FMA, Couto AN, Aerosa SVC, Montañés MCM. Grupos de convivência como suporte ao idoso na melhoria da saúde. Rev Bras Geriatr Gerontol. 2013;16(4):821-32. https://doi.org/10.1590/S1809-98232013000400016

19. Feng L, Nyunt MSZ, Feng L, Yap KB, Ng TP. Frailty predicts new and persistente depressive symptoms among communitydwelling older adults: finding from Singapore Longitudinal Aging Study. J Am Dir Assoc. 2014;15(1):76.e7-76.e12, https:// doi.org/10.1016/j.jamda.2013.10.001

20. Clares JWB, Guedes MVC, Silva LF, Nóbrega MML, Freitas MC. Subset of nursing diagnoses for the elderly in Primary Health Care. Rev Esc Enferm USP. 2016;50(2):270-76. https://doi.org/10.1590/S0080-623420160000200013

21. World Health Organization (WHO). Novel Coronavirus (2019-nCoV) technical guidance, 2020[Internet]. 2020 [cited 2020 Apr 04]. Available from: https://www.who.int/emergencies/diseases/novel-coronavirus-2019/technical-guidance

22. Andrade ABCA, Ferreira AA, Aguiar MJG. Conhecimento dos idoso sobre os sinais e sintomas da depressão. Saúde Redes. 2016;2(2):157-66. doi:10.18310/2446-4813.2016v2n2p157-166

23. FrankI VE. Um sentido para a vida: psicoterapia e humanismo. 13. ed. São Paulo: Ideias e Letras; 2005. 